\title{
Modifications in host cell structure and functions mediated by Tat intracellular expression are greatly dependent on the second exon María Rosa López-Huertas ${ }^{1}$, Sergio Callejas² ${ }^{2}$ David Abia ${ }^{3}$, Ana Dopazo², José Alcamí*1 and Mayte Coiras ${ }^{1}$
}

Address: ${ }^{1}$ AIDS Immunopathology Unit, Centro Nacional de Microbiología, Instituto de Salud Carlos III, Madrid, Spain, ${ }^{2}$ Genomics Unit, Centro Nacional de Investigaciones Cardiovasculares, Madrid, Spain and ${ }^{3}$ Bioinformatics Unit, Centro de Biología Molecular Severo Ochoa, CSIC-UAM, Madrid, Spain

* Corresponding author

from Frontiers of Retrovirology: Complex retroviruses, retroelements and their hosts Montpellier, France. 21-23 September 2009

Published: 24 September 2009

Retrovirology 2009, 6(Suppl 2):PI doi:10.II86/I742-4690-6-S2-PI

This abstract is available from: http://www.retrovirology.com/content/6/S2/PI

(c) 2009 López-Huertas et al; licensee BioMed Central Ltd.

\section{Background}

The human immunodeficiency virus type 1 (HIV-1) encodes the 110 amino acid-pleiotropic nuclear transcriptional activator Tat that is not only essential for efficient elongation of viral transcripts, but also regulates both cellular protein and gene expression. Tat encoding gene consists of two spliced exons separated in the HIV-1 genome by more than 2.300 nucleotides. First 72 residues at the $\mathrm{N}$ terminal encoded by the first exon appear to be sufficient for transcriptional activity, but the integrity of the coding second exon in most lentiviral genomes suggests that it should have some biological importance. In this work, we demonstrate that the second exon completes Tat function.

\section{Materials and methods}

The role of Tat in gene expression deregulation and functional biology of CD4+T lymphocytes was studied by analyzing the gene expression profile of Jurkat cells expressing intracellular full-length Tat (1-101aa) or a truncated form lacking the second exon (1-72aa) using whole human genome microarrays.

\section{Results}

More than 1.000 genes were deregulated in Jurkat Tat101, whereas less than 300 genes were deregulated in Jurkat Tat72 (q-value $<5 \%$; fold change $>2$ or $<-2$ ). Ontological analysis indicated that several functions were impaired mainly in Jurkat Tat 101 as cellular movement, growth and proliferation, cell-to-cell signaling, molecular transport, cell death, morphology, and T-cell activation. In accordance, biological and functional analyses proved that Tat101 expression induced changes in cell size and complexity, cytoskeletal structure and chemotaxis capacity, resistance to apoptosis, surface expression of adhesion molecules and receptors, and transcriptional activation. These alterations were attenuated or absent in Jurkat Tat72 cells. Furthermore, computational modeling showed that the absence of second exon severely reduced the C-terminus of Tat72, which would lessen the binding strength to its targets.

\section{Conclusion}

Full-length Tat produced dramatic structural changes and impaired essential functions in $\mathrm{CD} 4+\mathrm{T}$ cells, whereas Tat72 was less aggressive. Consequently, although first exon is transcriptionally autonomous, second exon appears to be necessary for triggering HIV-1 pathogenic events induced by Tat through the enhancement of the binding strength or affinity for its target sequences and protein partners. 